\title{
Integrin a7 knockdown suppresses cell proliferation, migration, invasion and endothelium- mesenchymal transformation in hepatocellular carcinoma
}

\section{Zhiyong Wu}

Department of General Surgery, The Central Hospital of Wuhan, Tongji Medical College, Huazhong University of Science and Technology

\section{Xiaoyu Kong}

Department of General Surgery, The Central Hospital of Wuhan, Huazhong Universty of Science and Technology

Zhihui Wang ( $\nabla$ huixin84002@163.com )

The Central Hospital of Wuhan, Tongji Medical College, Huazhong Universiy of Science and Technology

\section{Research article}

Keywords: Integrin a7, hepatocellular carcinoma, cell proliferation, apoptosis, endothelium-mesenchymal transformation

Posted Date: December 4th, 2019

DOI: https://doi.org/10.21203/rs.2.18036/v1

License: (c) (i) This work is licensed under a Creative Commons Attribution 4.0 International License. Read Full License 


\section{Abstract}

Background The aim was to investigate whether integrin a7 (ITGA7) influenced hepatocellular carcinoma (HCC) progression, and explore its effect on regulating endothelium-mesenchymal transformation (EMT).

Methods ITGA7 mRNA and protein expressions in human normal liver epithelial cell line and HCC cell lines were determined by reverse transcription polymerase chain reaction (RT-qPCR) and western blot. ITGA7 siRNA (ITGA7-KD group) and nonsense siRNA (control group) were transfected into Huh7 cells and SUN449 cells. After transfection, ITGA7 mRNA and protein expressions (RT-qPCR and western blot), cell proliferation (Cell Counting Kit-8), apoptosis (Annexin V/Propidium lodide assay), migration (Wound scratch assay) and invasion (Transwell assay) were determined. E-cadherin and a-SMA expressions (RTqPCR and western blot) were determined.

Results ITGA7 mRNA and protein expressions were increased in Li7, Huh7, SKHEP1 and SNU449 cells compared to THLE-3 cells. In both Huh7 and SNU449 cells, ITGA7 mRNA and protein expressions were decreased in ITGA7-KD group than control group after plasmids transfection, indicating the successful transfection. Then, cell proliferation was decreased at $48 \mathrm{~h}$ and $72 \mathrm{~h}$; cell apoptosis rate was increased at $48 \mathrm{~h}$; cell migration rate was reduced at $24 \mathrm{~h}$; cell invasive count was decreased at $24 \mathrm{~h}$ in ITGA7-KD group compared to control group. Furthermore, increased E-cadherin but decreased a-SMA mRNA and protein expressions were discovered in ITGA7-KD group than control group at $24 \mathrm{~h}$.

Conclusions ITGA7 knockdown suppresses HCC progression and inhibits EMT process in HCC, indicating that ITGA7 might be a potential novel treatment target for HCC therapy.

\section{Introduction}

Hepatocellular carcinoma (HCC) has been reported to be the top sixth commonly cancer and the fourth in causing cancer death all over the world in 2018 , which leads to more than 800,000 pupolation disgnosed as HCC and 700,000 population dead owning to HCC per year [1]. Surgical resection is the optimum selection in HCC patients, whereas there are just parts of them (who have small solitary tumors and very well-preserved liver function) could bear the trauma from this invasive operation and achieve good efficacy, but major of patients diagnosed as HCC at the first time have been in advanced, thereby losing their best time for surgical resection [2]. As for another curative treatment, liver transplantation is most conducive to HCC patients who are not good candidates for resection, while donor shortage and huge medical cost extremely limit its applicability [2]. Hence, deeply investigating molecular mechanisms of HCC progression is needful for the detection of novel and convincing therapeutic targets and the improvement of HCC prognosis.

Integrins, a kind of heterodimers consisting of an alpha and a beta subunit, participates in a broad spectrum of cellular processes, including cell survival, cell growth, cell invasion as well as cell migration [3]. Integrin a7 (ITGA7), belonging to the integrin family of adhesion molecules, is concerning in relevant cell-cell and cell-extracellular matrix interactions in a broad range of cellular processes [4]. According to 
previous studies, ITGA7 presents with oncogenic effect on the pathological processes of several carcinomas including glioblastoma, oesophageal squamous cell carcinoma (OSCC) as well as lung cancer [4-6]. Among which, ITGA7 promotes OSCC cell migration and invasion, meanwhile it regulates Ecadherin and a-SMA expressions (common markers of endothelium-mesenchymal transformation (EMT)), which indicates that ITGA7 promotes malignant cellular function and induces EMT in OSCC cells [5]. Whereas little information is found about its role in HCC. Thus, this study aimed to investigate whether ITGA7 influenced HCC progression, and explore its effect on regulating EMT.

\section{Methods}

\section{Cell culture}

Human normal liver epithelial cell lines THLE-3 and HCC cell lines SKHEP1 and SNU449 were purchased from American Type Culture Collect (ATCC) (VA, USA). HCC cell lines Li7 and Huh7 were purchased from RIKEN BioResource Research Center (Ibaraki, Japan). THLE-3 cells were cultured in a medium containing $90 \%$ BEGM (Lonza, USA) and $10 \%$ fetal bovine serum (FBS) (Gibco, USA). Li7 cells and SNU449 cells were cultured in a medium containing $90 \%$ Roswell Park Memorial Institute 1640 Medium (Gibco, USA) and $10 \%$ FBS (Gibco, USA). Huh7 cells were cultured in a medium containing $90 \%$ Dulbecco's Modified Eagle Medium (Gibco, USA) and $10 \%$ FBS (Gibco, USA). SKHEP1 cells were cultured in a medium containing 90 \% Eagle's Minimum Essential Medium (Gibco, USA) and 10 \% FBS (Gibco, USA). Cells were maintained in a humid incubator.

\section{Expression of ITGA7 in cell lines}

In THLE-3, Li7, Huh7, SKHEP1 and SNU449 cells, ITGA7 mRNA expression was determined by using reverse transcription polymerase chain reaction (RT-qPCR), and ITGA7 protein expression was determined by western blot. THLE-3 cells were served as control in the RT-qPCR.

\section{ITGA7 plasmids construction and transfection}

Small interfering RNA (siRNA) was employed to knock down ITGA7. ITGA7 siRNA and nonsense siRNA were designed and synthesized by Guangzhou RiboBio Co., Ltd (Guangzhou, China). ITGA7 siRNA and nonsense siRNA were transfected into Huh7 cells and SUN449 cells using Lipofectamine 2000 (Invitrogen, USA) based on the manufacturer's instructions. Subsequently, cells transfected with ITAG7 siRNA were named as ITGA7-KD cells, correspondingly, cells transfected with nonsense siRNA were marked as control cells. At 24 hours (h) after transfection, the mRNA and protein expressions of ITGA7 were determined by RT-qPCR and western blot; the cell apoptosis was detected by Annexin V/Propidium lodide (AV/PI) assay; and the cell migration ability and invasive ability were determined by wound scratch assay and transwell assay. Meanwhile, the cell viability was assessed by using Cell Counting Kit-8 (CCK- 
8) at $0 \mathrm{~h}, 24 \mathrm{~h}, 48 \mathrm{~h}$ and $72 \mathrm{~h}$, respectively. Furthermore, the mRNA and protein expressions of E-cadherin and a-SMA, which were regulated by ITGA7, were determined by RT-qPCR and western blot at $24 \mathrm{~h}$ after transfection.

\section{RT-qPCR}

After cells digested by $0.25 \%$ Trypsin (Gibco, USA), TRIzol (Invitrogen, USA) was utilized for the extraction of total RNA. 1 ug RNA was reversely transcribed to cDNA by using PrimeScript ${ }^{\text {TM }}$ RT reagent Kit (Takara, Japan). TB Green ${ }^{\text {TM }}$ Fast qPCR Mix (Takara, Japan) was utilized for qPCR. The PCR amplification was performed: $95^{\circ} \mathrm{C}$ for $5 \mathrm{~min}$, followed by 40 cycles of $95^{\circ} \mathrm{C}$ for $5 \mathrm{~s}, 61^{\circ} \mathrm{C}$ for $30 \mathrm{~s}$. The result was calculated using $2^{-\triangle \triangle \mathrm{Ct}}$. The sequences of primers were shown in Table 1. For an internal reference, glyceraldehyde-phosphate dehydrogenase (GAPDH) was selected.

\section{Western Blot}

Pierce $^{\mathrm{TM}}$ BCA Protein Assay Kit (Thermo, USA) was utilized to measure the concentration of protein after total protein extraction by using RIPA Lysis and Extraction Buffer (Thermo, USA). The $20 \mu \mathrm{g}$ protein sample was fractionated by NuPAGE ${ }^{\text {TM }} 4-20 \%$ Tris-Acetate Midi Protein Gels (Thermo, USA), and transfected to Polyvinylidene Fluoride (PVDF) membrane. And then, the membrane was blocked by $5 \%$ skim milk for $2 \mathrm{~h}$, and incubated with the primary antibody at $4{ }^{\circ} \mathrm{C}$ overnight. The secondary antibody was incubated ( $1 \mathrm{~h}$ at room temperature). The chemiluminescence of blots was performed by using Pierce ${ }^{\mathrm{TM}}$ ECL Plus Western Blotting Substrate (Invitrogen, USA) and then exposed to X-ray film (Kodak, USA) following treatment. GAPDH was considered as the internal reference. Antibodies used in Western Blot were listed in Table 2.

\section{CCK-8}

After adding $10 \mathrm{ul} \mathrm{CCK8}$ and 90 ul RPMI 1640 medium into each plate, cells were incubated at $37^{\circ} \mathrm{C}$ with $5 \% \mathrm{CO}_{2}$ by using CCK-8 (Dojindo, Japan). The optical density (OD) value was using by Microplate reader (Biotek, USA).

\section{$\mathrm{AV} / \mathrm{PI}$}

Cells were digested with pancreatin, and then washed with phosphate buffer saline (PBS). After suspending in $100 \mathrm{ul} \mathrm{Biding} \mathrm{Buffer} \mathrm{and} \mathrm{adding} 5 \mathrm{ul} \mathrm{AV}$ and $5 \mathrm{ul} \mathrm{Pl}$, cells were incubated in the dark) by using Annexin V-FITC Apoptosis Detection Kit (Sigma, USA). 


\section{Wound scratch assay}

Cells were cultured until $80 \%$ growth confluence, and then were scraped with a sterile pipette tip to inflicting adherent cells gaps (which was set as $0 \mathrm{~h}$ ). Subsequently, the cells were incubated. Cells were observed at $\mathrm{O} \mathrm{h}$ and $24 \mathrm{~h}$ by using Microscope (Nikon, Japan).

\section{Transwell assay}

After coated Matrigel basement membrane matrix (BD, USA) on the upper site of transwell Chamber (Costar, USA), cells were seeded in the upper site of chamber. After incubating and wiping out the upper cells, cells on the lower sider of the chamber were fixed using formaldehyde (Sigma, USA). After stained with $0.5 \%$ crystal violet (Sigma, USA) for 15 min, the invasive cells count of each well was calculated by the average invasive cells count of 5 fields in each well, which was observed under $100 \mathrm{X}$ magnification of a Microscope (Olympus, Japan).

\section{Statistical analysis}

Statistical analysis and graph plotting were performed on the GraphPad Prism 7.02 software (GraphPad Software Inc., USA). Data were shown as mean and standard deviation (SD). Comparison was performed by the unpaired $\mathrm{t}$ test, while the multiple comparison was used by the Dunnett $\mathrm{t}$ test. * meant $P$ value $<0.05$; $* *$ meant $P$ value $<0.01 ; * \star *$ meant $P$ value $<0.001$. $P<0.05$ meant significance.

\section{Results}

\section{ITGA7 expression in HCC cell lines and human normal liver epithelial cell line}

mRNA expression of ITGA7 was higher in HCC cell lines including Li7 $(P<0.01)$, Huh7 $(P<0.001)$, SKHEP1 $(P<0.01)$ and SNU449 $(P<0.05)$ cells compared to human normal liver epithelial cell lines THLE-3 cells (Figure 1A). In addition, ITGA7 protein expression was elevated in HCC cell lines including Li7, Huh7, SKHEP1 and SNU449 cells compared to human normal liver epithelial cell lines THLE-3 cells as well (Figure 1B).

\section{ITGA7 expression after transfection}

After plasmids transfection, both mRNA (Figure 2A) and protein (Figure 2C) expressions of ITGA7 were decreased in ITGA7-KD group than that in control group $(P<0.001)$ in Huh7 cells. In SNU449 cells, both 
mRNA (Figure 2B) and protein (Figure 2D) expressions of ITGA7 also were lower in ITGA7-KD group than that in control group $(P<0.001)$.

\section{Cell proliferation and apoptosis after transfection}

We subsequently investigated whether ITGA7 influenced cell proliferation and apoptosis in Huh7 and SNU449 cells. In Huh7 cells, cell proliferation was decreased in ITGA7-KD group at $48 \mathrm{~h}(P<0.05)$ as well as $72 \mathrm{~h}(P<0.01)$ than that in control group (Figure $3 \mathrm{~A})$, meanwhile, cell apoptosis rate at $48 \mathrm{~h}$ after transfection was increased in ITGA7-KD group $(P<0.01)$ compared to control group (Figure 3C, 3E). In SNU449 cells, cell proliferation was reduced in ITGA7-KD group at $48 \mathrm{~h}(P<0.05)$ as well as $72 \mathrm{~h}(P<0.05)$ than that in control group (Figure 3B), and cell apoptosis rate at $48 \mathrm{~h}$ after transfection was enhanced in ITGA7-KD group than that in control group $(P<0.01)$ (Figure 3D, 3F).

\section{Cell migration after transfection}

After that, we explored whether ITGA7 influenced cell migration in Huh7 and SNU449 cells. In Huh7 cells, wound scratch assay disclosed that migration rate (at $24 \mathrm{~h}$ after transfection) was lower in ITGA7-KD group compared to control group $(P<0.01)$ (Figure 4A, 4C). In SNU449 cells, migration rate (at $24 \mathrm{~h}$ after transfection) was also lower in ITGA7-KD group than that in control group $(P<0.001)$ (Figure 4B, 4D).

\section{Cell invasion after transfection}

In order to investigate whether ITGA7 influenced cell invasion in Huh7 and SNU449 cells, we performed transwell assay. In Huh7 cells, invasion cell number was decreased in ITGA7-KD group than that in control group $(P<0.01)$ at $24 \mathrm{~h}$ after transfection (Figure 5A, 5C). In SNU449 cells, invasion cell number was also lower in ITGA7-KD group than that in control group $(P<0.01)$ at $24 \mathrm{~h}$ after transfection as well (Figure 5B, 5D).

\section{EMT markers after transfection}

So as to deeply explore the effect of ITGA7 on EMT in HCC pathogenesis, we detected EMT markers including E-cadherin and a-SMA expressions in Huh7 and SNU449 cells after transfection. In Huh7 cells, E-cadherin mRNA $(P<0.05)$ and protein expressions (Figure 6A, 6E) were increased, but a-SMA mRNA $(P<0.05)$ and protein expressions (Figure 6C, 6E) were decreased in ITGA7-KD group than that in control group at $24 \mathrm{~h}$ after transfection. In SNU449 cells, E-cadherin mRNA $(P<0.01)$ and protein expressions 
(Figure 6B, 6F) were enhanced, but a-SMA mRNA $(P<0.05)$ and protein expressions (Figure 6D, 6F) were reduced in ITGA7-KD group than that in control group at $24 \mathrm{~h}$ after transfection as well.

\section{Discussion}

Integrin family is one of transmembrane protein receptors attaching cells to the extracellular matrix and binding ligands secreted by other cells $[7,8]$. Serving as one of family members of integrins, ITGA7 have been determined as a vital role in tumor propagation and CSCs properties regulation $[5,9]$. Recent studies reveal that ITGA7 is highly expressed in various cancer cells including OSCC as well as mesothelioma [5, 10]. Although these previous studies have detected high ITGA7 expression in various cancer cells, its role in HCC is still unclear. Therefore, we discovered that ITGA7 was overexpressed in HCC cell lines (including Li7, Huh7, SKHEP1 and SNU449 cells) compared to human normal liver epithelial cell line THLE-3 cells, which might be caused by that ITGA7, as its oncogenic effect on tumor progression in other cancers, could regulate several genes or pathways (including focal adhesion kinase (FAK)/ serine protein kinase (AKT)-zinc finger E-box Binding Homeobox 1 (ZEB1) signaling way) to promote cell growth and metastasis, subsequently contributing to the malignant transformation of HCC $[5,11]$. Thus, ITGA7 was overexpressed in HCC cells.

According to several previous studies, ITGA7 is involved in the pathological progress of different carcinomas through affecting cell activities, including cell migration as well as cell invasion. For example, ITGA7 interacting with laminin-induced outside-in signaling contributes to glioblastoma stem-like cells growth and invasion [11]. Furthermore, ITGA7 binding with S100P could activate FAK/ AKT-ZEB1 signaling way to induce lung cancer cell migration and invasion [6]. Meanwhile, ITGA7 also has been confirmed to be related to the stemness of cancer cells. In a later study, ITGA7 drives stemness of OSCC cells through FAK/ mitogen-activated protein kinase (MAPK)/ extracellular signal-regulated kinase (ERK) signaling ways, subsequently induced tumorigenicity and metastasis of OSCC [5]. However, several lines of evidence disclose a tumor suppressor role of ITGA7 in various malignancies. For example, ITGA7 appears to activate cyclin-dependent kinase inhibitor 3 (CDKN3) and Rac GTPase-activating protein 1 (RACGAP1) expressions to inhibit cell motility and decrease metastases in HCC cells [12]. Taken these previous studies together, although a few studies have been performed to explore the role of ITGA7 in difference carcinomas, there are still some contradictions. More importantly, little information is known about its role in HCC. So as to investigate the effect of ITGA7 on cell activities in HCC, we explored its effect on HCC cell activities, we discovered that ITGA7 knockdown decreased cell proliferation, migration and invasion, but increased apoptosis in HCC cells, which suggested that ITGA7 knockdown might suppress the function of HCC. The possible explanations were as follows: (1) ITGA7, like its cancerogenic effect on tumor progression in lung cancer, might interact with S100P to promoting FAK/ AKT-ZEB1 signaling way to enhance $\mathrm{HCC}$ cell proliferation, migration and invasion, thereby contributing to tumor progression of HCC [5]. (2) ITGA7, like its promotive influence on tumor progression in glioblastoma, might be binging with laminin-induced outside-in signaling to accelerate HCC cells growth and invasion, thereby leading to tumor progression of HCC [11]. (3) ITGA7 might attach cells to the extracellular matrix and interact with ligands secreted by other cells to activate HCC cell invasion and migration, 
subsequently promoting tumor progression of HCC. (4) ITGA7 might regulate CDKN3 (that has responsible to dephosphorylate tyrosine residues of different cyclin-dependent kinases (CDKs) and repress cell cycle progression in yeast and mammalian cells) to increase HCC cell invasion and motility, subsequently accelerating tumor progression in HCC [12-14]. (5) ITGA7 might influence RACGAP1 to increase cell growth, enhance cell motility and promote tumor metastases. Taken together, ITGA7 knockdown suppressed tumor progression of $\operatorname{HCC}[12,15]$.

Limited information about whether ITGA7 plays an important role in EMT transition to ultimately promote tumor metastasis is found $[5,16,17]$. Just one previous study discloses that ITGA7 promotes OSCC cell migration and invasion, more importantly, ITGA7 also induces EMT in OSCC [5]. Meanwhile, EMT is not only a well-coordinated process touched off by multiple signaling pathways during embryonic development, but also a kind of pathological characteristic in neoplasia and fibrosis, which has been considered as an essential regulator linked to tumor progression and tumor metastasis through accelerating cancer cell invasion and dissemination to distant organs [18-21]. In order to explore whether ITGA7 influenced EMT in HCC, we further detected E-cadherin and a-SMA expressions, and we found that ITGA7 knockdown increased E-cadherin expression while decreased a-SMA expression in HCC cells. In sum up, ITGA7 knockdown might repress EMT process in HCC.

\section{Conclusion}

In conclusion, ITGA7 knockdown suppresses HCC cell proliferation, migration, invasion and EMT, but promotes apoptosis. These data indicate that ITGA7 might be a novel and convincing treatment target for HCC therapy.

\section{Declarations}

\section{Ethics approval and consent to participate}

This study did not require an ethical board approval because it did not contain human or animal trials.

\section{Consent for publication}

Not applicable.

\section{Availability of data and material}

All data generated or analysed during this study are included in this published article.

\section{Competing interests}


The authors declare that they have no competing interests.

\section{Funding}

Not applicable.

\section{Authors' contributions}

ZYW and $X Y K$ designed and coordinated the study, made substantial contributions to the analysis, and drafted the manuscript. ZYW, XYK and XHZ performed statistical analysis. ZYW, XYK and XHZ participated in data collection and interpretation. All authors draft the manuscript. All authors have read and approved the final manuscript.

\section{Acknowledgements}

Not applicable.

\section{References}

1.Bray F, Ferlay J, Soerjomataram I, Siegel RL, Torre LA, Jemal A. Global cancer statistics 2018:

GLOBOCAN estimates of incidence and mortality worldwide for 36 cancers in 185 countries. CA Cancer J Clin. 2018; 68:394-424.

2.Forner A, Llovet JM, Bruix J. Hepatocellular carcinoma. Lancet. 2012; 379:1245-55.

3.Desgrosellier JS, Cheresh DA. Integrins in cancer: biological implications and therapeutic opportunities. Nat Rev Cancer. 2010; 10:9-22.

4.Carrasco-Garcia E, Auzmendi-Iriarte J, Matheu A. Integrin alpha7: a novel promising target in glioblastoma stem cells. Stem Cell Investig. 2018; 5:2.

5.Ming XY, Fu L, Zhang LY, Qin YR, Cao TT, Chan KW et al. Integrin alpha7 is a functional cancer stem cell surface marker in oesophageal squamous cell carcinoma. Nat Commun. 2016; 7:13568.

6.Hsu YL, Hung JY, Liang YY, Lin YS, Tsai MJ, Chou SH et al. S100P interacts with integrin alpha7 and increases cancer cell migration and invasion in lung cancer. Oncotarget. 2015; 6:29585-98.

7.Hynes RO. Integrins: bidirectional, allosteric signaling machines. Cell. 2002; 110:673-87.

8.Tan LZ, Song Y, Nelson J, Yu YP, Luo JH. Integrin alpha7 binds tissue inhibitor of metalloproteinase 3 to suppress growth of prostate cancer cells. Am J Pathol. 2013; 183:831-40. 
9.Nunes AM, Barraza-Flores P, Smith CR, Burkin DJ. Integrin alpha7: a major driver and therapeutic target for glioblastoma malignancy. Stem Cell Investig. 2017; 4:97.

10.Burkin DJ, Fontelonga TM. Mesothelioma cells breaking bad: loss of integrin alpha7 promotes cell motility and poor clinical outcomes in patients. J Pathol. 2015; 237:282-4.

11. Haas TL, Sciuto MR, Brunetto L, Valvo C, Signore M, Fiori ME et al. Integrin alpha7 Is a Functional Marker and Potential Therapeutic Target in Glioblastoma. Cell Stem Cell. 2017; 21:35-50 e9.

12.Ren B, Yu YP, Tseng GC, Wu C, Chen K, Rao UN et al. Analysis of integrin alpha7 mutations in prostate cancer, liver cancer, glioblastoma multiforme, and leiomyosarcoma. J Natl Cancer Inst. 2007; 99:868-80.

13.Gyuris J, Golemis E, Chertkov H, Brent R. Cdi1, a human G1 and S phase protein phosphatase that associates with Cdk2. Cell. 1993; 75:791-803.

14.Hannon GJ, Casso D, Beach D. KAP: a dual specificity phosphatase that interacts with cyclindependent kinases. Proc Natl Acad Sci U S A. 1994; 91:1731-5.

15.Kawashima T, Hirose K, Satoh T, Kaneko A, Ikeda Y, Kaziro Y et al. MgcRacGAP is involved in the control of growth and differentiation of hematopoietic cells. Blood. 2000; 96:2116-24.

16.Brabletz T. EMT and MET in metastasis: where are the cancer stem cells? Cancer Cell. 2012; 22:699701.

17.Mani SA, Guo W, Liao MJ, Eaton EN, Ayyanan A, Zhou AY et al. The epithelial-mesenchymal transition generates cells with properties of stem cells. Cell. 2008; 133:704-15.

18.Zou J, Li H, Huang Q, Liu X, Qi X, Wang Y et al. Dopamine-induced SULT1A3/4 promotes EMT and cancer stemness in hepatocellular carcinoma. Tumour Biol. 2017; 39:1010428317719272.

19.Santamaria PG, Moreno-Bueno G, Portillo F, Cano A. EMT: Present and future in clinical oncology. Mol Oncol. 2017; 11:718-38.

20.Nistico P, Bissell MJ, Radisky DC. Epithelial-mesenchymal transition: general principles and pathological relevance with special emphasis on the role of matrix metalloproteinases. Cold Spring Harb Perspect Biol. 2012; 4.

21.Gaianigo N, Melisi D, Carbone C. EMT and Treatment Resistance in Pancreatic Cancer. Cancers (Basel). 2017; 9.

\section{Tables}

Table 1. Primers applied in RT-qPCR 


\begin{tabular}{lll}
\hline Gene & Forward Primer (5'-3') & Reverse Primer (5'-3') \\
\hline ITGA7 & GCCACTCTGCCTGTCCAATG & GGAGGTGCTAAGGATGAGGTAGA \\
E-cadherin & TGATTCTGCTGCTCTTGCTGTT & CCTCTTCTCCGCCTCCTTCTT \\
$\alpha$-SMA & CATTCACGAGACCACCTACAACAG & CGCCGATCCACACCGAGTAT \\
GAPDH & GACCACAGTCCATGCCATCAC & ACGCCTGCTTCACCACCTT \\
\hline
\end{tabular}

RT-qPCR: reverse transcription polymerase chain reaction

Table 2. Antibodies applied in western blot

\begin{tabular}{lll}
\hline Antibody & Company & Dilution \\
\hline Primary Antibody & & \\
ITGA7 mouse mAb & Santa Cruz (USA) & $1: 1000$ \\
E-cadherin mouse mAb & Santa Cruz (USA) & $1: 1000$ \\
a-SMA mouse mAb & Santa Cruz (USA) & $1: 1000$ \\
GAPDH mouse mAb & Santa Cruz (USA) & $1: 2000$ \\
Secondary Antibody & & \\
Goat Anti-mouse IgG-HRP & Santa Cruz (USA) & $1: 5000$ \\
\hline
\end{tabular}

\section{Figures}

A

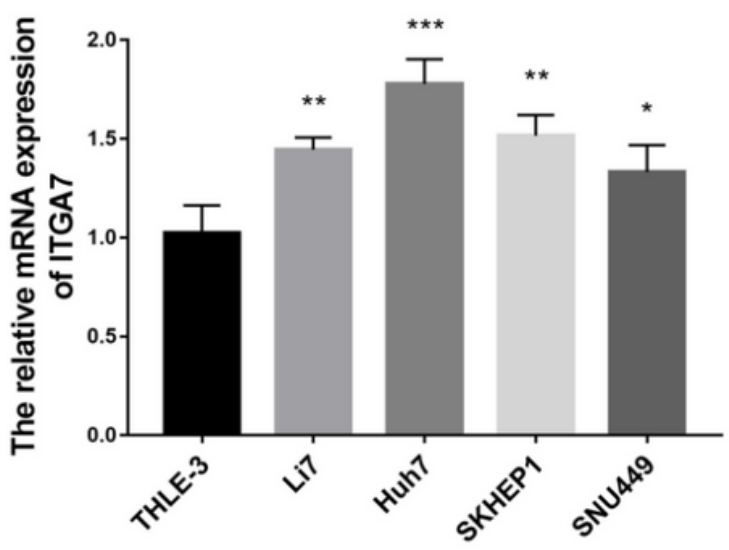

B

$\begin{array}{llll}\text { THLE-3 Li7 } & \text { Huh7 } & \text { SKHEP1 SNU449 }\end{array}$

ITGA7

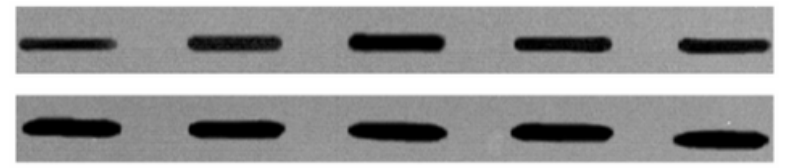

\section{Figure 1}

The expression of ITGA7. A: ITGA7 mRNA expression in HCC cell lines and human normal liver epithelial cell line; B: ITGA7 protein expression in HCC cell lines and human normal liver epithelial cell line. ITGA7: 
integrin 7; GAPDH: glyceraldehyde-phosphate dehydrogenase.

Huh7 cells

A

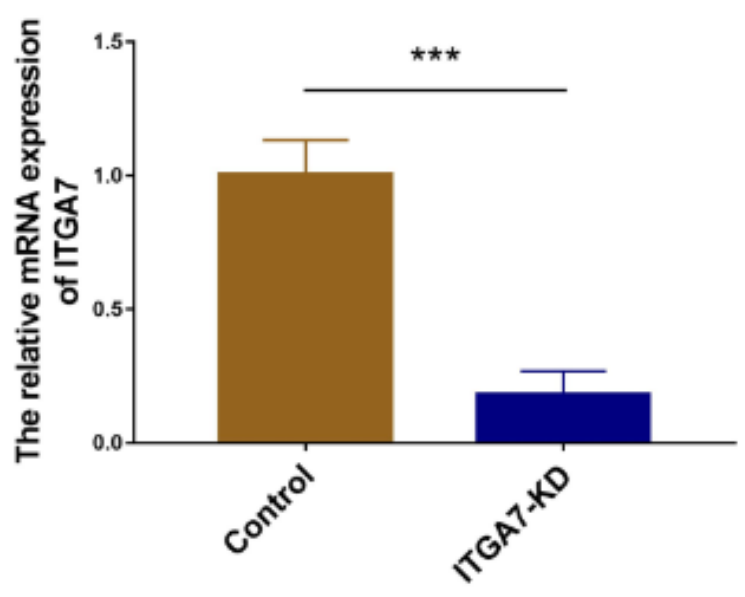

C
B

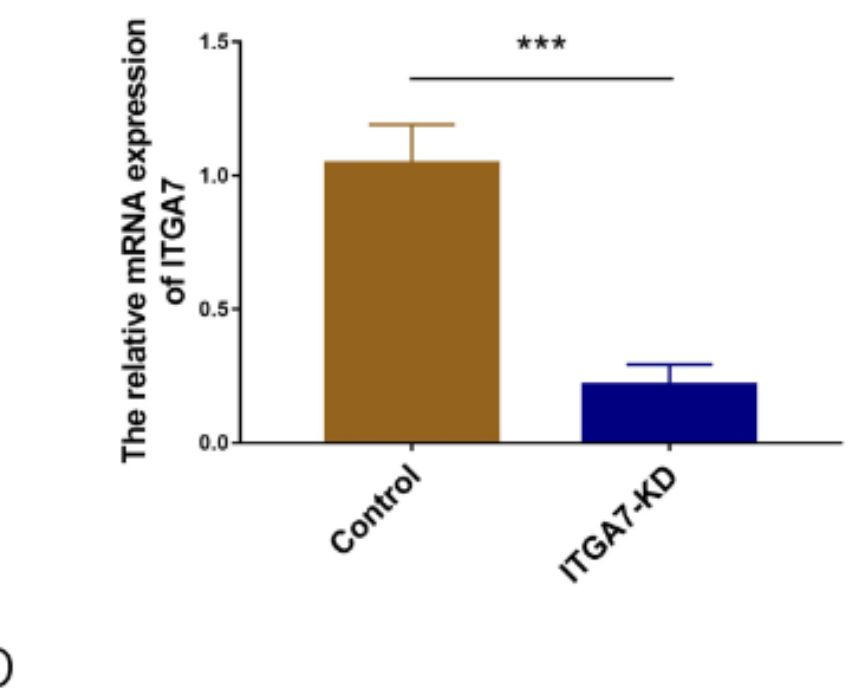

D

$B$

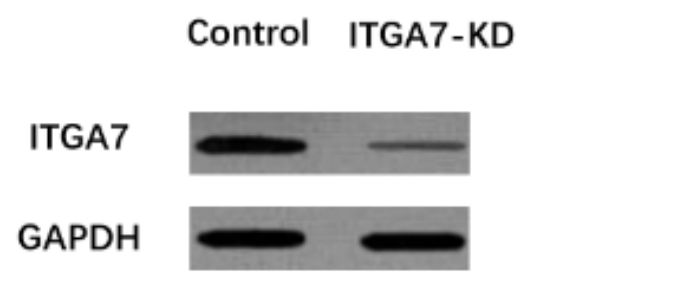

Control ITGA7-KD

ITGA7

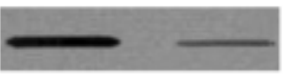

GAPDH

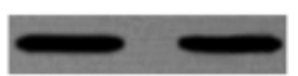

Figure 2

The expression of ITGA7 after transfection by ITGA7 siRNA plasmids. A, B: ITGA7 mRNA expression in Huh7 cells and SNU449 cells; C, D: ITGA7 protein expression in Huh7 cells and SNU449 cells. ITGA7: integrin 7; GAPDH: glyceraldehyde-phosphate dehydrogenase. 
A

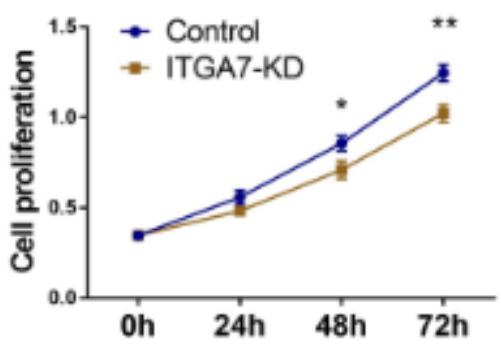

$\mathrm{C}$

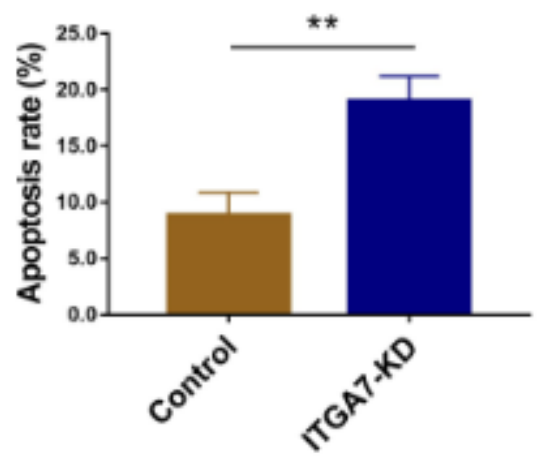

$E$

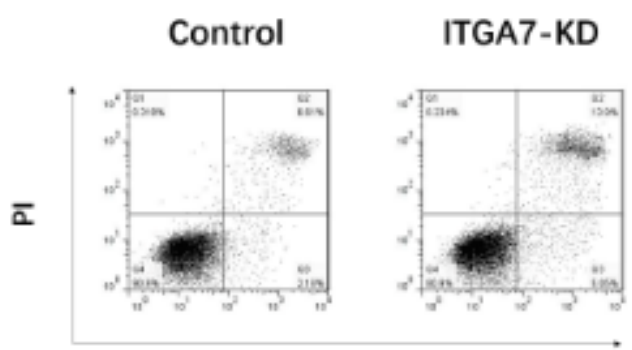

AV
B

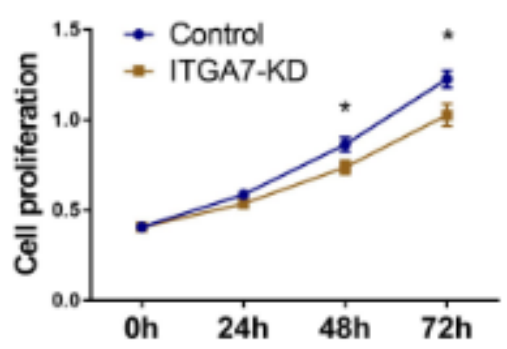

D

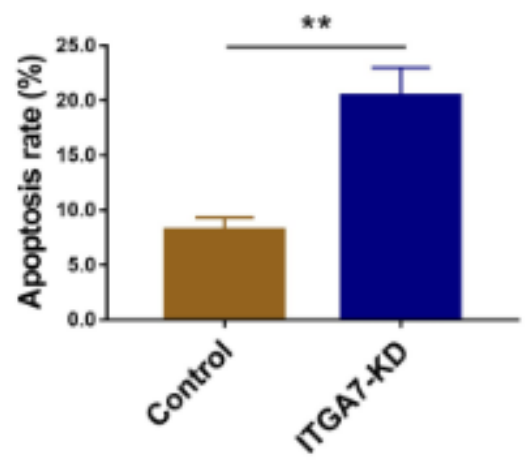

F

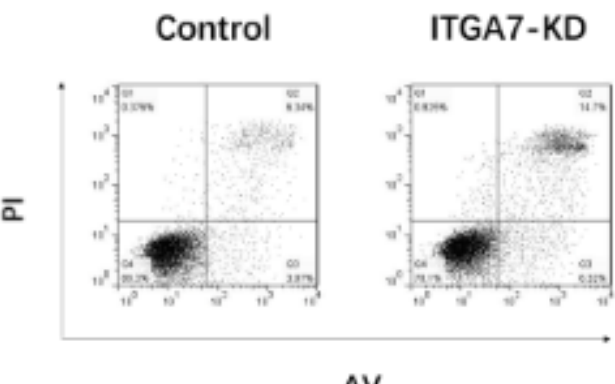

Figure 3

Cell proliferation and apoptosis. A, B: Cell proliferation after transfection in Huh7 cells and SNU449 cells; C, E: Cell apoptosis after transfection in Huh7 cells; D, F: Cell apoptosis after transfection in SNU449 cells. ITGA7: integrin 7. 


\section{Huh7 cells}

A

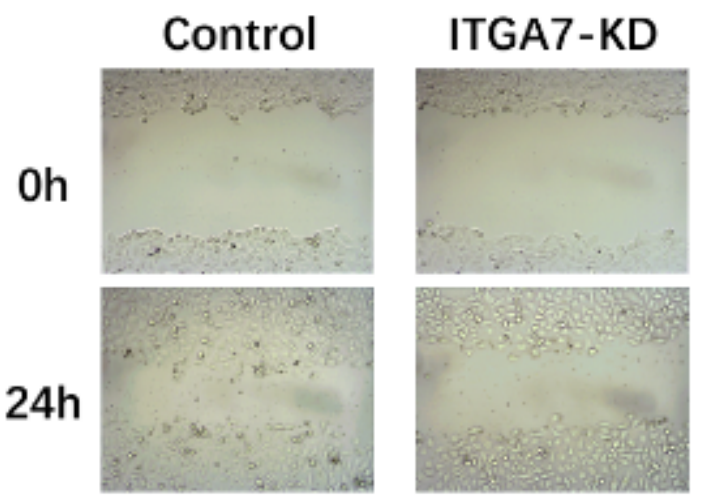

C

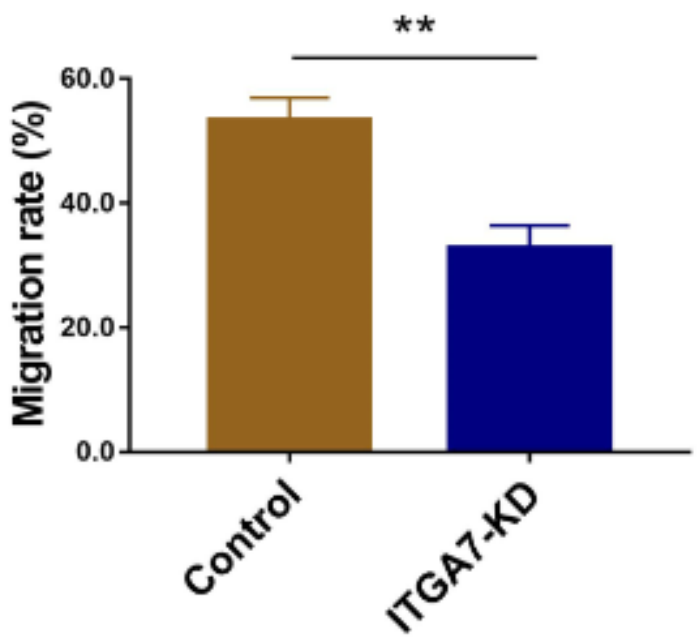

SNU449 cells

B

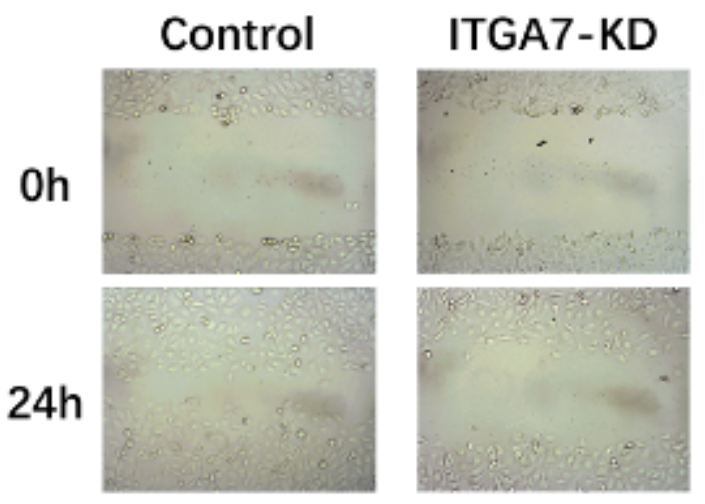

D

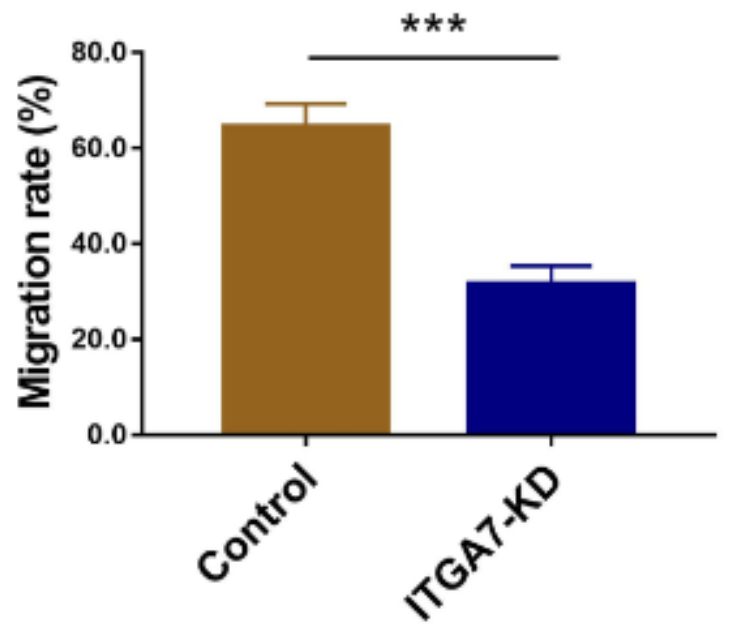

Figure 4

Cell migration. A, C: Cell migration after transfection in Huh7 cells; B, D: Cell migration after transfection in SNU449 cells. ITGA7: integrin 7. 
A

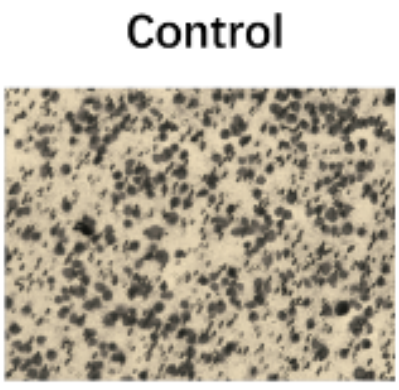

Control

ITGA7-KD

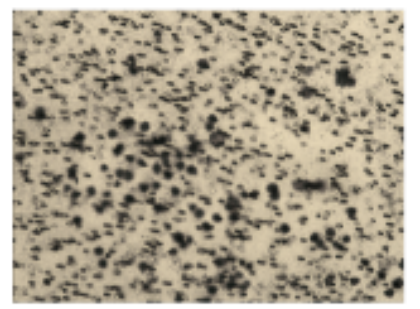

C

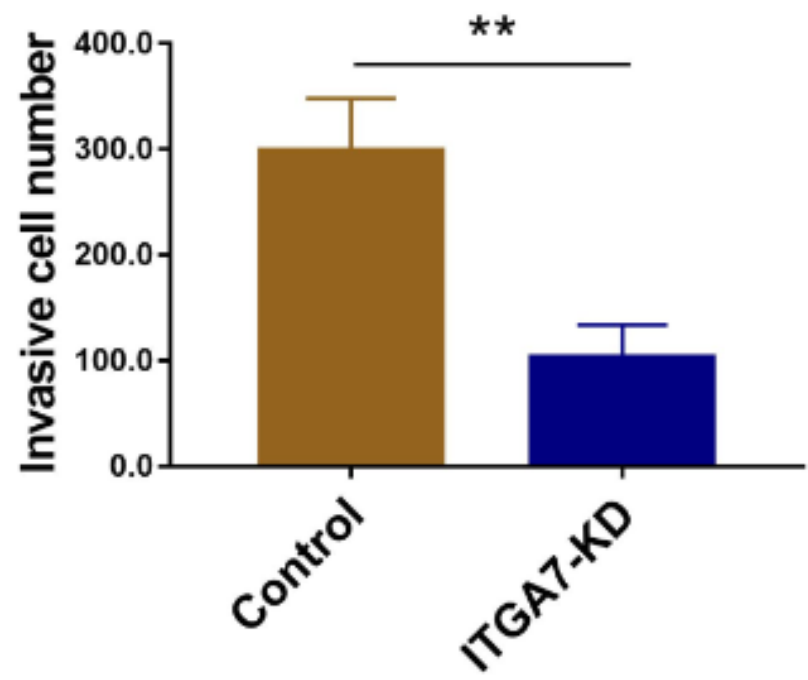

B
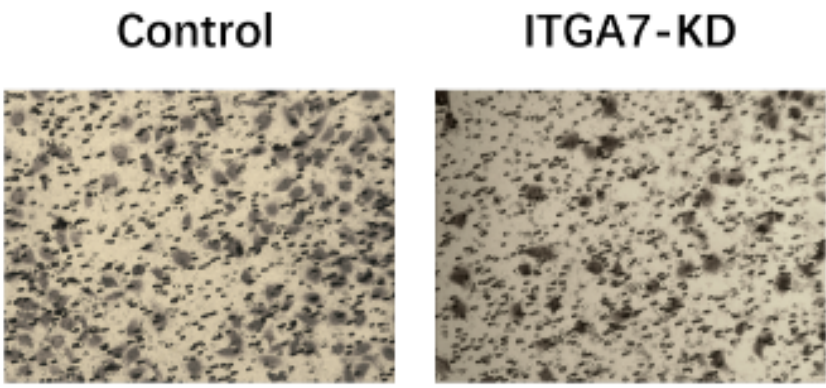

D

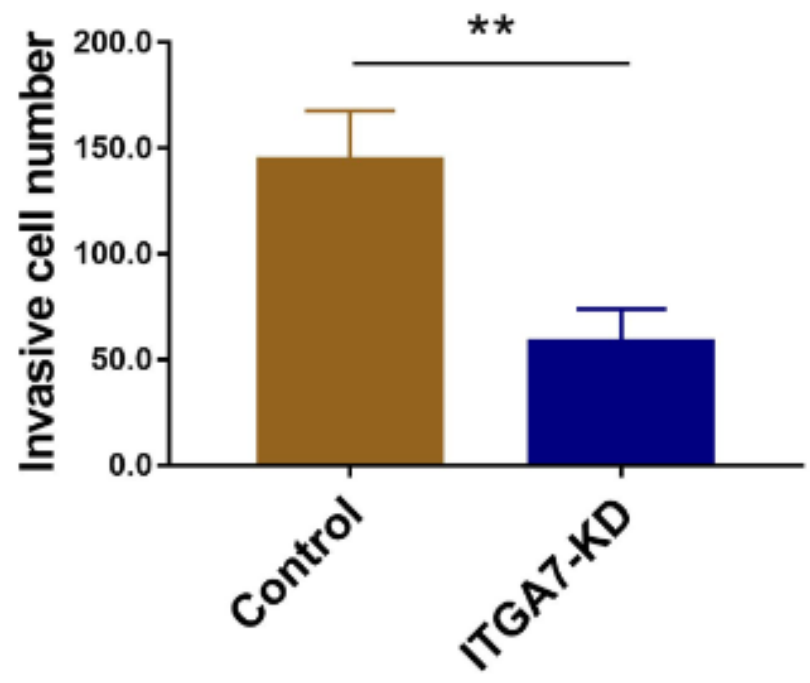

Figure 5

Cell invasion. A, C: Cell invasion after transfection in Huh7 cells; B, D: Cell invasion after transfection in SNU449 cells. ITGA7: integrin 7. 
Huh7 cells

A

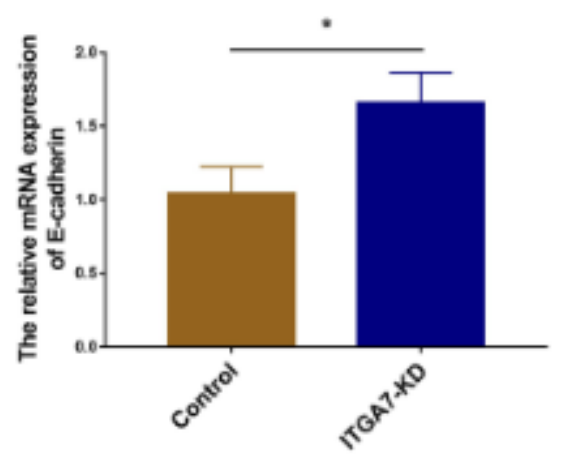

$\mathrm{C}$

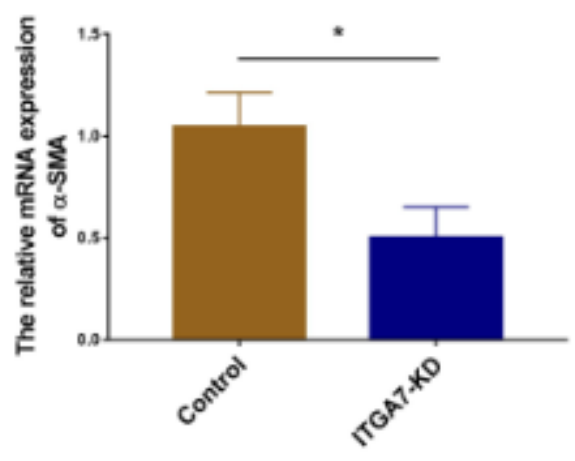

E

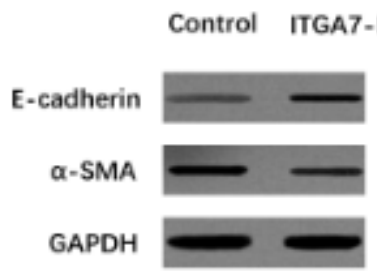

B

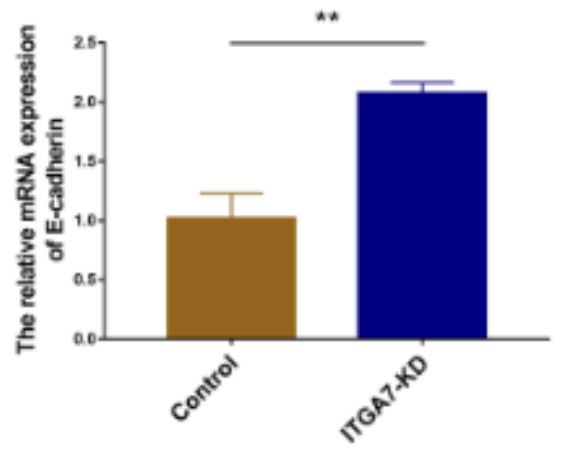

D

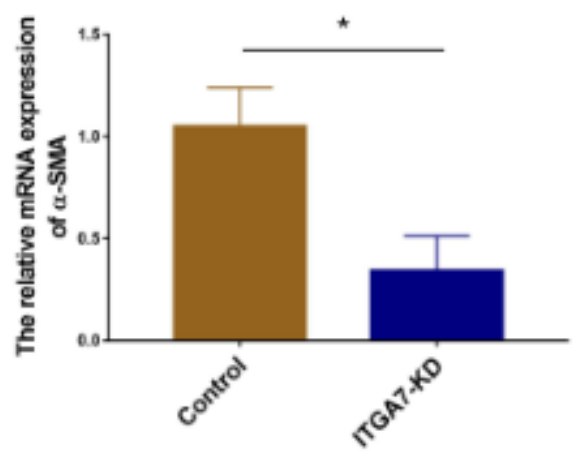

$\mathrm{F}$

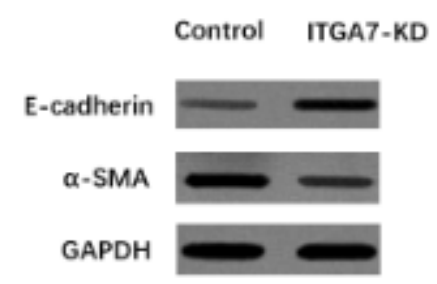

Figure 6

The effect of ITGA7 on E-cadherin and a-SMA expressions. A, B: E-cadherin mRNA expression after transfection in Huh7 cells and SNU449 cells; C: a-SMA mRNA expression after transfection in Huh7 cells and SNU449 cells; E, F: E-cadherin and a-SMA protein expressions after transfection in Huh7 cells and SNU449 cells. ITGA7: integrin 7; GAPDH: glyceraldehyde-phosphate dehydrogenase. 